\title{
Factores de riesgo perinatal, signos neurológicos blandos y lenguaje en edad preescolar
}

\author{
Patricia Torres Morales, * Dora Elizabeth Granados Ramos**
}

\begin{abstract}
RESUMEN
Introducción: Los factores de riesgo perinatal son características que pueden presentarse en un periodo de 28 días previos y 28 días posteriores al parto, que incrementan la probabilidad de estructurar una secuela del desarrollo. Los signos neurológicos blandos son indicadores de alteraciones cerebrales mínimas o retardo en la maduración cerebral; ambos factores pueden contribuir en la presencia de dificultades de lenguaje, siendo importante su detección e intervención oportuna. Objetivo: Conocer la relación entre factores de riesgo, signos neurológicos blandos y lenguaje en edad preescolar. Se evaluó a 22 preescolares de un jardín de niños de Xalapa, Veracruz, México (11 de sexo femenino y 11 de sexo masculino) con edad promedio de 5 años 8 meses (DE \pm 4 meses). Material y métodos: Estudio observacional, prospectivo y descriptivo, se aplicaron las pruebas de lenguaje y signos neurológicos blandos de la Evaluación Neuropsicológica Infantil (ENI) y la Subescala Verbal de la Escala de Inteligencia Wechsler para los Niveles Preescolar y Primario en Español (WPPSI por sus siglas en inglés). Mediante una historia clínica y datos de egreso del hospital se documentaron los factores de riesgo perinatales, calificándose con los criterios de CIMIGEN. Resultados: Siete casos tuvieron riesgo perinatal bajo, nueve tuvieron medio y seis alto. Once niños no tuvieron dificultades de lenguaje, cuatro presentaron dificultades severas, tres presentaron moderadas y cuatro leves. Se realizó una T de Student al comparar presencia/ausencia de dificultades de lenguaje con el riesgo perinatal y los signos neurológicos blandos y una ANOVA de una vía al comparar los puntajes de ENI y WPPSI con el riesgo perinatal y los signos neurológicos blandos, con diferencias significativas de $\mathrm{p}<0.05$ al comparar presencia/ausencia de dificultades con marcha, articulación y signos neurológicos blandos totales. Conclusiones: Los factores de riesgo perinatal pueden condicionar la presencia de signos neurológicos y dificultades del lenguaje en la edad preescolar. Es fundamental la prevención y detección temprana de los factores de riesgo así como la intervención oportuna ante la presencia de signos neurológicos blandos a fin de evitar la estructuración de dificultades en los procesos de aprendizaje como el lenguaje.
\end{abstract}

Palabras clave: Riesgo perinatal, signos neurológicos blandos, lenguaje.

\section{Perinatal risk factors, neurological soft signs and language in preschool}

\begin{abstract}
Introduction: Perinatal risk factors are characteristics present that can occur in a period of 28 days before and 28 days after birth, which increases the possibility of structuring a development sequel. Soft neurological signs are indicators of minimal brain abnormalities or delay in brain maturation; both factors may contribute to the presence of language difficulties, still important detection and intervention. Objective: The aim of the investigation was to determine the relationship between risk factors, neurological soft signs and language in preschool. We evaluated to 22 preschool kindergartens from Xalapa,
\end{abstract}

* Universidad Veracruzana, Doctorado en Investigaciones Cerebrales.

** Universidad Veracruzana, Facultad de Psicología-Xalapa, Laboratorio de Psicobiología.

Correspondencia: Patricia Torres Morales. E-mail: torresmoralespatricia@yahoo.com.mx

Este artículo puede ser consultado en versión completa en http://www.medigraphic.com/enfermerianeurologica 
Veracruz, Mexico (11 female and 11 male) with a mean age of 5 years 8 months (SD \pm 4 months). Material and methods: Observational, prospective, descriptive, tests were applied Language and Neurological Soft Signs of Neuropsychological Assessment of Children (ENI) and the Subscale Verbal of the Wechsler Preschool and Primary Scale of Intelligence in Spanish (WPPSI). By a medical history and hospital discharge data were documented perinatal risk factors, qualifying with CIMIGEN criteria. Results: Seven cases had low perinatal risk, nine medium risk, and six high risk. Eleven children had no language difficulties, four had severe difficulties, three moderate and four mild. We performed a Student's T-test to compare the presence/absence of language difficulties with perinatal risk and neurological soft signs and one-way ANOVA to compare ENI-WPPSI scores with perinatal risk and soft neurological signs, with differences significant at $\mathrm{p}<0.05$ when comparing presence/absence of language difficulties with march, articulation and total neurological soft signs. Conclusions: Perinatal risk factors may determine the presence of neurological signs and language difficulties in the preschool years. Is essential the prevention and early detection of risk factors as well as early intervention in the presence of soft neurological signs in order to avoid structuring difficulties in learning processes such as language.

Key words: Perinatal risk, neurological soft signs, language.

\section{INTRODUCCIÓN}

$\mathrm{L}$ os primeros cinco años de vida se consideran como los más relevantes en el desarrollo del lenguaje, ya que en ellos se deben consolidar los aspectos fonológicos, semánticos, sintácticos y pragmáticos que le permitirán al niño desarrollar un lenguaje adecuado, sin la presencia de dificultades de expresión o comprensión. ${ }^{1}$ En la etapa preescolar, que en México abarca de 3 a 5 años de edad, se fortalecen y consolidan los aprendizajes necesarios para la adquisición de conocimientos más complejos durante la etapa escolar, entre éstos, la adquisición de la lectura y la escritura. ${ }^{2}$

Durante la etapa preescolar pueden observarse dificultades en el aprendizaje y la adquisición del lenguaje, pudiendo ser resultado de la presencia de factores de riesgo perinatal y signos neurológicos blandos que no han sido documentados a profundidad y que pueden terminar por limitar el desarrollo íntegro del niño. ${ }^{3}$

En el periodo perinatal, comprendido entre los 28 días previos y los 28 días posteriores al nacimiento, se encuentran factores de riesgo definidos como características o circunstancias observables o detectables en un individuo de algún hecho que se asocia con la probabilidad de padecer, desarrollar o estar expuesto a un proceso mórbido y a su subsecuente secuela. ${ }^{4}$ Estos factores pueden ser de tipo biológico, ambiental, comportamental o de estilo de vida, relacionados con la atención a la salud, socioculturales y socioeconómicos, considerándose tanto los riesgos maternos como los del niño en la vida intrauterina y del recién nacido. La interacción de factores de riesgo biológicos, sumados a otros derivados del medio social y ambiental, aumenta el efecto aislado de cada uno de estos factores de riesgo. ${ }^{4}$

Según el número de factores presentados, el riesgo se clasifica en tres niveles: bajo, medio y alto. En el riesgo bajo se ubican los casos en los que no se encuentran presentes factores que pongan en riesgo la salud de la madre o el niño, donde ambos tiene condiciones de normalidad estables; en el riesgo medio se ubican los casos en los que existen factores de riesgo que, de no atenderse con eficacia, pueden agravarse y generar daño a la madre, al feto o al recién nacido; y en el riesgo alto, se encuentran los casos donde existen factores de riesgo con alta probabilidad de daño al feto, a la madre o al recién nacido. ${ }^{5,6}$

Los signos neurológicos blandos, considerados como indicadores de alteraciones cerebrales mínimas o de retardo en la maduración cerebral ${ }^{7,8}$ pueden contribuir, junto a los antecedentes de riesgo perinatal, a la estructuración de dificultades en el aprendizaje preescolar y en áreas tan importantes como el lenguaje.

En la edad preescolar pueden presentarse dificultades en la articulación de ciertos fonemas, tales como las dislalias, donde no existen patologías del sistema nervioso central que originan dificultades articulatorias, y que producen severos retrasos en la comunicación oral del niño y el desarrollo de aprendizajes como la lectoescritura. La presencia de dificultades del lenguaje debe ser considerada desde etapas tempranas, para evitar secuelas mayores que obstaculicen el desarrollo del niño. ${ }^{1}$

El objetivo de la presente investigación fue conocer la relación entre factores de riesgo, signos neurológicos blandos y características del lenguaje del niño preescolar.

\section{MATERIAL Y MÉTODOS}

Se realizó un estudio observacional, prospectivo y descriptivo con 22 niños preescolares inscritos en un jardín de niños oficial de la ciudad de Xalapa, Veracruz, México, con una edad promedio de cinco años ocho meses ( $\mathrm{DE} \pm 4$ meses); 11 de sexo femenino y 11 de sexo masculino, con un nivel socioeconómico bajo. 
A cada sujeto se le aplicó la Evaluación Neuropsicológica Infantil (ENI) y la Escala de Inteligencia Wechsler para los niveles Preescolar y Primario en Español (WPPSI por sus siglas en inglés). ${ }^{10}$ Para esta investigación, se consideraron los resultados de las subpruebas correspondientes a lenguaje (repetición, expresión y comprensión) y signos neurológicos blandos de la ENI, y los resultados de la subescala verbal de WPPSI para obtener el cociente intelectual verbal (CIV) de cada sujeto.

Los puntajes de las subpruebas de lenguaje se calificaron mediante los rangos de la ENI como: $>75$, por arriba del promedio (AP); de 75 a 26, promedio (P); de 25 a 11, promedio bajo (PB); de 10 a 3, bajo (B), y $<2$ como extremadamente bajo (EB).

Se contó el número de signos neurológicos blandos presentado por cada niño, valorándose aspectos como la capacidad de marcha (marcha sobre una línea recta, en puntas y brincar con ambos pies), el agarre del lápiz (tipo de pinza al tomar el lápiz), la articulación, (repetición de fonemas y detección de los que están mal articulados), la agudeza visual (uso o no de anteojos), la agudeza auditiva (detección de sonidos débiles realizados con la fricción de los dedos de la mano), la discriminación derecha-izquierda (en él mismo y en otras personas), el seguimiento visual (valorando la presencia de asimetrías y/o nistagmo), la extinción táctil (identificación alternada del tacto ligero en sus manos), la extinción visual (detección del campo visual derecho e izquierdo), la disdiadococinesis (capacidad para realizar movimientos alternados de las manos), movimientos de oposición digital (presencia de movimientos lentos, asimetrías o movimientos asociados al tocar los dedos de ambas manos con el dedo pulgar) y la lateralidad manual (diestra, zurda o mixta).

El CIV se calificó mediante los rangos de puntaje de la escala WPPSI en: < 130 como muy superior (MS); de 129 a 120 como superior (S); de 119 a 110 como promedio alto (PA); de 109 a 90 como promedio (P); de 89 a 80 como promedio bajo (PB); de 79 a 70 como límite (L), y $<69$ como muy bajo (MB).

Mediante una historia clínica y la hoja de egreso hospitalaria de cada niño, se documentó con las madres de los niños la presencia de factores de riesgo pre, peri y postnatales; los factores de riesgo perinatal encontrados se analizaron con base en la Evaluación Inicial de Riesgo Perinatal (PREVIGen II) ${ }^{11}$ que considera los factores de riesgo relacionados con la madre y el niño.

Los indicadores de riesgo perinatal de la madre son el nivel socioeconómico, la edad al momento del parto, la escolaridad, la estatura, el estado civil, la paridad, dificultades en el parto anterior, número de abortos, presencia de enfermedades como diabetes, hipertensión, desnutrición, toxoplasmosis, rubéola, citomegalovirus, herpes, sífilis,
SIDA u otras enfermedades de tipo infeccioso, presencia de tabaquismo, alcoholismo o consumo de drogas lícitas y/o ilícitas, la presión arterial durante el embarazo y el parto, el aumento de peso total durante el embarazo y la salud de hijos previos al nacimiento (bajo peso, malformaciones, nacimiento prematuro o muerte neonatal).

Los indicadores de riesgo perinatal del niño se documentan según sus características en el periodo de vida intrauterina y de recién nacido. En la vida intrauterina, los indicadores fueron los movimientos fetales, la presencia/ausencia de hemorragia genital, la amenaza de aborto, las características del líquido amniótico, la presencia/ausencia de malformaciones, la duración del trabajo de parto, el tipo de nacimiento, la posición fetal y la presencia/ausencia de trauma fetal. En el recién nacido, los indicadores fueron el peso al nacimiento, la calificación APGAR al minuto y a los cinco minutos, la edad gestacional, si requirió maniobras de reanimación, si presentó insuficiencia respiratoria, hipoxia, ictericia y/o cianosis, las características de su llanto, la presencia/ausencia de convulsiones al nacimiento, las características de la succión-deglución, las características de la fontanela, la presencia/ausencia de opistótonos, el tono muscular, la prensión y las características de la búsqueda de objetos y rostros del recién nacido.

A los factores de riesgo perinatal, se les asignó un nivel de riesgo según lo establecido en los criterios del Centro de Investigaciones Materno-Infantil (CIMIGen). ${ }^{11}$ Los indicadores de la madre y el niño se conjuntaron para obtener los niveles de riesgo de cada niño, clasificándose con dos indicadores para riesgo alto, tres para riesgo medio y cinco para riesgo bajo.

Se realizó una T de Student para comparar estadísticamente el número de signos neurológicos blandos según la presencia/ausencia de dificultades de lenguaje, y una ANOVA de una vía para comparar el nivel de riesgo perinatal y los signos neurológicos blandos según los puntajes de lenguaje (repetición, expresión y comprensión) de la ENI y el CIV del WPPSI, usando el programa estadístico JMP 8.

\section{RESULTADOS}

En relación con el análisis de los factores de riesgo, se encontró que el 27\% de los participantes (seis casos) presentaron riesgo perinatal alto, el $41 \%$ (nueve casos) tuvieron un riesgo perinatal medio y el $32 \%$ (siete casos) tuvieron un riesgo perinatal bajo.

Los indicadores que tuvieron mayor frecuencia en el nivel de riesgo alto y medio fueron un nivel socioeconómico muy bajo y bajo. La edad de la madre durante el embarazo fue mayor de 30 años o menor de 20. La duración del trabajo de parto fue igual o mayor a nueve horas. El nacimiento 
fue por cesárea programada o urgente. Hubo presencia de trauma fetal de uno a dos abortos espontáneos anteriores y amenaza de aborto durante el embarazo. El aumento total de peso durante el embarazo fue de 15 a 20 kilogramos y el peso del niño al nacer fue de 3,700 a 4,000 gramos.

Según el sexo de los participantes, en el nivel de riesgo alto se ubicó un 9\% de las niñas (dos casos) y 18\% de los niños (cuatro casos); en el nivel de riesgo medio, un 23\% de las niñas (cinco casos) y $18 \%$ de los niños (cuatro casos); y en el nivel de riesgo bajo, $18 \%$ de las niñas (cuatro casos) y $14 \%$ de los niños (tres casos) (Figura 1).

En cuanto a las características del lenguaje, el 50\% de los niños (once casos) no presentaron dificultades de lenguaje; el 50\% (11 casos) tuvieron dificultades de lenguaje. De los participantes sin dificultades de lenguaje, tres casos reportaron un riesgo perinatal alto y cuatro casos, respectivamente, presentaron riesgo medio y riesgo bajo; de los participantes con dificultades del lenguaje, tres casos reportaron riesgo alto, cinco casos presentaron riesgo medio y tres casos, riesgo bajo (Figura 2).

Todos los participantes tuvieron un CIV promedio. En repetición del lenguaje, el 18\% tuvo puntajes bajos (PB, B y $\mathrm{EB})$, el $41 \%$ puntajes promedio (P) y el $41 \%$ restante, obtuvo puntajes superiores al promedio (AP); en expresión, el $91 \%$ tuvo puntajes promedio $(\mathrm{P})$ y el $9 \%$ puntajes superiores al promedio (AP); y en comprensión, el 5\% tuvo puntajes bajos (EB), el $73 \%$ puntajes promedio (P) y el $23 \%$ puntajes superiores al promedio (AP).

Los signos neurológicos que se presentaron con más frecuencia fueron dificultades en la marcha, problemas para discriminar derecha e izquierda, dificultades para realizar movimientos coordinados de dedos (oposición digital) y manos (disdiadococinesis) y problemas articulatorios (Cuadro I).

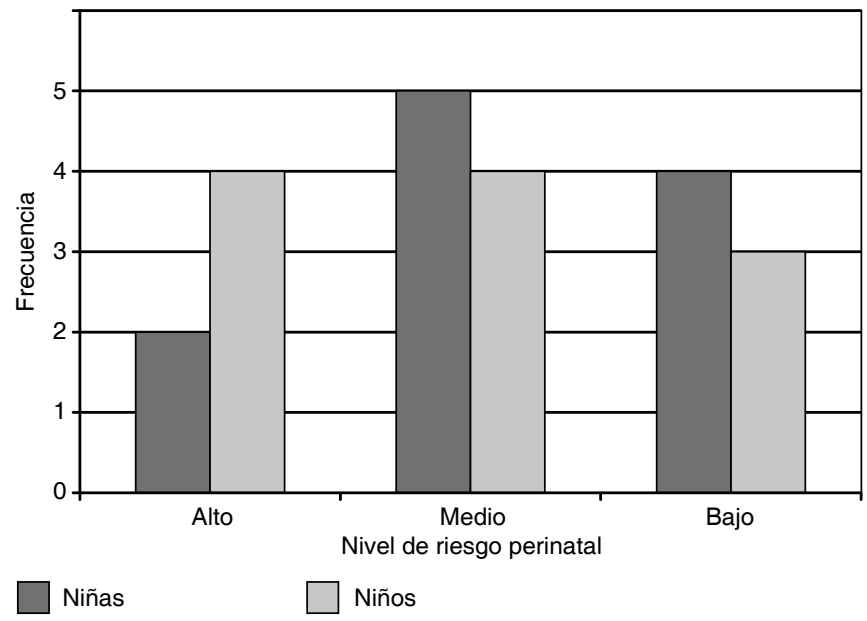

Figura 1. Distribución de los niños por nivel de riesgo perinatal y sexo.
Las dificultades del lenguaje encontradas en este estudio fueron de tipo articulatorias, principalmente, dificultades para la pronunciación de los fonemas $/ \mathrm{r} / \mathrm{y} / \mathrm{rr} / \mathrm{al}$ inicio, en medio o al final de las palabras, al modificar el sonido por uno semejante como "pela" por "pera" o "tambol" por "tambor". En la pronunciación de /r/ en grupos consonánticos donde le antecedía o precedía otra consonante, siendo sustituido el sonido de $/ \mathrm{r} /$ por $/ \mathrm{l} / \mathrm{en}$ los grupos consonánticos /br/, /pr/, /gr/, /fr/, /tr/, /dr/ y /kr/, por ejemplo, dijo "blazo" por "brazo", "pleso" por "preso", "tigle" por "tigre". En los grupos consonánticos /rk/, /rb/ y /rd/ omitieron el sonido de /r/ como en "ábol" por "árbol", y "godo" por "gordo".

Además, se observaron omisiones del sonido /l/ en los grupos consonánticos /fl/, /kl/, /gl/, /pl/, /bl/, /ls/ y /ld/, por ejemplo, dijo "gobo" por "globo" y "pátano" por "plátano". Al realizar T de Student, se observaron diferencias estadísticamente significativas al comparar presencia/ausencia de dificultades del lenguaje con la marcha $(\mathrm{F}=8.36 \mathrm{p}<0.009)$, la articulación $(\mathrm{F}=12.08 \mathrm{p}<.002)$ y el total de signos neurológicos blandos presentados $(\mathrm{F}=20.66 \mathrm{p}<0.002)$, en donde el grupo de niños con dificultades de lenguaje presentaron

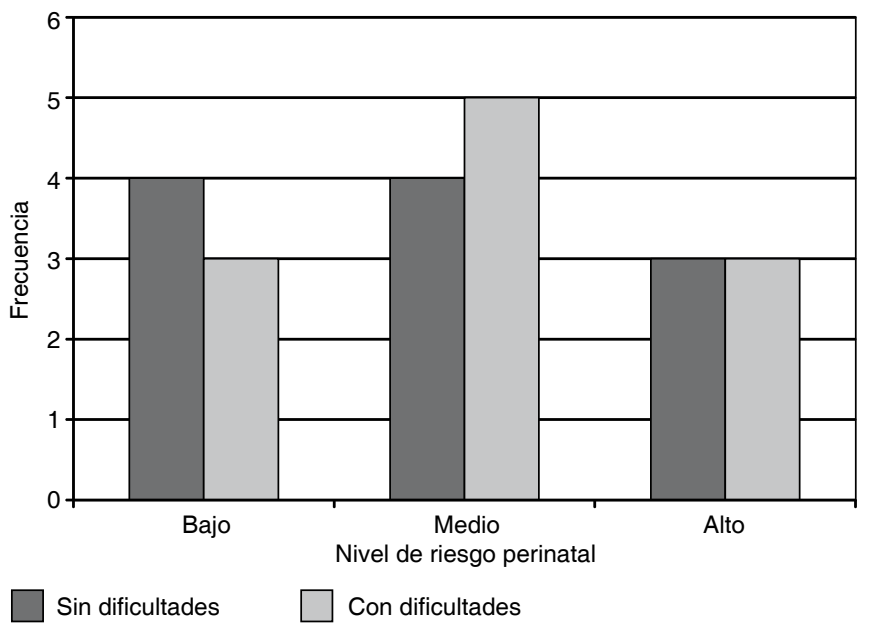

Figura 2. Distribución de los niños por nivel de riesgo perinatal y lenguaje.

Cuadro I. Frecuencia de los signos neurológicos blandos según el nivel de riesgo perinatal presentado por los participantes.

\begin{tabular}{lccc}
\hline & \multicolumn{3}{c}{ Riesgo Perinatal } \\
\hline Signos Neurológicos Blandos & Bajo & Medio & Alto \\
\hline Discriminación derecha-izquierda & 7 & 9 & 5 \\
Marcha & 3 & 8 & 3 \\
Oposición digital & 3 & 7 & 4 \\
Disdiadococinesis & 2 & 4 & 4 \\
Articulación & 2 & 4 & 3 \\
Seguimiento Visual & 0 & 2 & 1 \\
\hline
\end{tabular}


mayor número de signos neurológicos blandos totales, así como mayor número de dificultades de motricidad gruesa en la marcha y articulatorias.

En el análisis ANOVA de una vía, se encontraron diferencias estadísticamente significativas al comparar los grupos por percentiles en repetición del lenguaje de ENI con el número total de signos neurológicos blandos $(\mathrm{F}=16.33$ $\mathrm{p}<0.01)$ y la articulación $(\mathrm{F}=31.53 \mathrm{p}<0.01)$, donde los participantes con puntajes EB y B presentaron el mayor número de signos neurológicos blandos y problemas en la articulación fonémica.

\section{DISCUSIÓN}

Durante la etapa preescolar, es fundamental un adecuado desarrollo del lenguaje, ya que éste favorecerá la adquisición de nuevos aprendizajes tales como la lectoescritura en la edad escolar; sin embargo, la presencia de factores de riesgo perinatal podría dificultar el aprendizaje del lenguaje.

Los niños con factores de riesgo durante la gestación y al momento del nacimiento son más propensos a presentar dificultades en la adquisición del lenguaje, e incluso a padecer algún trastorno subsecuente del lenguaje. ${ }^{12}$ Las dificultades del lenguaje pueden estar asociadas con el nivel de riesgo perinatal, ya que mientras mayor sea el número de factores de riesgo, mayores serán las implicaciones cognitivas que dificulten la adquisición de procesos aprendidos como el lenguaje. ${ }^{5}$ En nuestros resultados encontramos que el $68 \%$ de los niños reportaron un nivel de factores de riesgo perinatal medio a alto, lo que nos indica la gran presencia de factores de riesgo perinatal que podrían estar afectando el desarrollo de los niños preescolares.

Los datos obtenidos en esta investigación nos permitieron conocer cuál es la relación entre los factores de riesgo, los signos neurológicos blandos y las características del lenguaje de los niños evaluados, ya que se observaron diferencias en el lenguaje de los niños según su nivel de riesgo perinatal y los signos neurológicos blandos de cada caso, por lo que se podría considerar que el objetivo se cumplió satisfactoriamente.

En el estudio, encontramos que la edad de la madre en cuanto a ser mayor de 30 o menor de 20 años, la presencia de trauma fetal, abortos anteriores o amenaza de aborto fueron los factores más reportadas por las madres de los niños con dificultades de lenguaje, coincidiendo con investigaciones previas que han encontrado asociación de las variables mencionadas con la presencia de trastornos del lenguaje. ${ }^{13}$ Se ha documentado que otras complicaciones obstétricas influyen en el deterioro del lenguaje, como el caso de la privación del oxígeno en periodos perinatales y al momen- to de nacimiento, donde niños con antecedentes de asfixia o hipoxia presentaron problemas del habla posteriores. ${ }^{14}$ Asimismo, se ha reportado que el bajo peso al nacer, si bien no es un factor de riesgo específico en los casos con problemas del lenguaje, sí representa un factor de riesgo implicado en dificultades cognitivas, como el retraso mental, o problemas perceptuales y motores, ${ }^{15}$ en nuestros casos, al contrario de un bajo peso, encontramos que prevaleció un peso mayor a los 3,500 gramos al nacer; en la literatura se describe que el sobrepeso del recién nacido está asociado con factores como el tabaquismo de la madre y malos hábitos al dormir. ${ }^{16}$

Otro de los factores de riesgo reportado en nuestros resultados fue el nivel socioeconómico bajo, el cual puede influir en el desarrollo del niño, ya que se ha reportado que escasos recursos económicos y/o de bajo nivel educativo de la madre se asocian a un nivel de riesgo más alto, que el de los niños de madres con un nivel socioeconómico de medio a alto y con un mayor número de años de estudio. ${ }^{17}$

La prevención de un trastorno específico del lenguaje implica identificar los factores de riesgo perinatal de los niños antes de que se estructure la dificultad, y así no sólo se prevendrían las dificultades de lenguaje sino también, el impacto que éstas pueden tener en otras áreas del aprendizaje como la lectura y escritura, ya que está demostrado que el aprendizaje de la lectoescritura está relacionado directamente con la expresión y comprensión del lenguaje. ${ }^{12}$

En cuanto a las evaluaciones neurológicas como parte de la valoración del desarrollo en la etapa preescolar, los signos neurológicos blandos, al ser indicadores de retardo en la maduración cerebral ${ }^{6}$ proporcionan una herramienta dentro de la detección de características que pudieran impedir la adquisición y desarrollo adecuado del lenguaje. Se ha reportado que el trastorno específico del lenguaje puede ocurrir en niños sin evidencia de algún tipo de discapacidad neurológica; sin embargo, esto no implica que no existan irregularidades del sistema nervioso central. ${ }^{12}$ La presencia de pequeñas irregularidades en el desarrollo y la maduración del sistema nervioso pueden influir en la presencia de dificultades del lenguaje, coincidiendo con nuestros resultados, donde todos los participantes presentaron signos neurológicos. Nosotros describimos que las dificultades para caminar sobre una línea recta se observaron en los niños con problemas de lenguaje, dichas dificultades podrían relacionarse con dificultades en las coordinaciones motoras necesarias para la escritura. ${ }^{17}$

La identificación de los factores de riesgo prenatales, perinatales y postnatales es una herramienta que permite conocer cómo impactan en el desarrollo cognitivo del niño. La intervención temprana inmediata a la detección 
de los factores de riesgo presentes en cada caso permitirá prevenir la estructuración de secuelas como los trastornos del lenguaje. ${ }^{18}$ Identificar a los niños con riesgo perinatal en el momento del nacimiento y conocer la presencia de signos neurológicos blandos a lo largo de la vida del niño, cuando el lenguaje no está presente y conforme se vaya adquiriendo, desarrollando y consolidando, puede facilitar la prevención de las dificultades del lenguaje en etapas preescolares. ${ }^{12}$

\section{CONCLUSIONES}

Factores de riesgo perinatal pueden condicionar la presencia de un mayor número de signos neurológicos blandos e influir en dificultades del lenguaje presentadas durante la edad preescolar.

Es fundamental la prevención y detección temprana de los factores de riesgo y la intervención oportuna ante su detección y la presencia de signos neurológicos blandos en etapas neonatales y durante los primeros años de vida, a fin de evitar que se estructuren dificultades en los procesos de aprendizaje, como el lenguaje.

Las dificultades de lenguaje deben ser detectadas en las primeras etapas de adquisición de éste, para que en etapas escolares no se vea mermado el aprendizaje de la lectoescritura; asimismo deben sugerirse a los padres de familia estrategias para estimular el lenguaje de sus hijos.

Para continuar esta investigación, se deberán analizar posteriormente los factores de riesgo pre y postnatales, así como trabajar con grupos de igual número de niños con distinta severidad en las dificultades de lenguaje para conocer las características propias de cada grupo.

\section{AGRADECIMIENTOS}

Agradecemos al CONACyT por la beca doctoral número $515188 / 289332$ en investigaciones cerebrales.

\section{BIBLIOGRAFÍA}

1. Azcoaga JE, Bello JA, Citrinovitz J, Derman B, Frutos WM. Los retardos del lenguaje en el niño. Barcelona: Editorial Paidós; 1981.

2. Guzmán GC, Saucedo RC. La investigación sobre alumnos en México: dimensiones y tendencias principales (1992-2002). En: Ducoing WP. Sujetos actores y procesos de formación. México: COMIE; 2005.

3. Bishop DVM, Genetic and environmental risk for specific language impairment in children. International Congress Series 2003; 1254: 225-245

4. Castillo C. Manual sobre el enfoque de riesgo en la atención materno infantil. Washington DC: OPS; 1999.

5. Rivera A, Hernández-Rodríguez L. Importancia del programa de reanimación neonatal. México: Universidad Nacional Autónoma de México UNAM; 2006.

6. Vargas-García C. Sistema de detección y evaluación de riesgo perinatal México: Centro de Investigación Materno Infantil CIMIGen; 2007.

7. Spreen O, Risser AT, Edgell D. Developmental neuropsychology. New York: Oxford University Press; 1995.

8. Patankar VC, Sangle JP, Shah HR, Dave M, Kamath RM, Neurological soft signs in children with attention deficit hyperactivity disorder. Indian J Psychiatry. 2012; 54 (2): 159-165.

9. Matute E, Rosselli M, Ardila A, Ostrosky-Solís F. Evaluación Neuropsicológica Infantil (ENI): Manual de aplicación. México: Editorial El Manual Moderno; 2007.

10. Wechsler, D. Escala de Inteligencia Wechsler para los Niveles Preescolar y Primario en Español WPPSI. México: Editorial El Manual Moderno; 1981.

11. Centro de Investigaciones Materno-Infantil. Sistema para Detección y Evaluación de Riesgo Perinatal; 1991

12. Stanton-Chapman TL, Chapman DA, Bainbridge NL, Scott KG, Identification of early risk factors for language impairment. Res Dev Disabil. 2002; 23: 390-405.

13. Sylvestre A, Mérette C, Language delay in severely neglected children: acumulitive or specific effect of risk factors? Child Abuse Negl. 2010 34: 414-428.

14. D'Souza SW, McCartney E, Nolan M, Taylor IG, Hearing, speech, and language in survivors of severe perinatal asphyxia. Arch Dis Child. 1991; 56: $245-252$.

15. Tomblin JB, Hardy J, Hein H, Predicting poor communication status in preschool children using risk factors present at birth. J Speech Hear Res. 1991; 34: 1096-1105.

16. Shi Y, De Groh M, Morrison H, Perinatal and early childhood factors for overweight and obesity in young Canadian children. Can J Public Health. 2013; 104 (1): 69-74.

17. Tomblin JB, Smith E, Zhang X, Epidemiology of specific language impairment: prenatal and perinatal risk factors. J Commun Disord. 1997; 30: $325-344$

18. Nation K, Developmental language disorders. Psychiatry. 2008; 7 (6): 266-269. 